Revista Matemática Universitária, vol. 1, 2021

ISSN: 2675-5254 — DOI:10.21711/26755254/rmu20213

\title{
QUANDO OS MÉTODOS DE EULER E DE NEWTON COINCIDEM
}

\author{
JOSÉ CLAUDINEI FERREIRA
}

Resumo: Este texto é dedicado ao estudo do uso de problemas de otimização e equações diferenciais para a dedução de métodos numéricos para a determinação de zeros de funções. Em particular, tratamos de estimativas sobre convergência exponencial para o método de Euler, em duas abordagens distintas, sendo que em uma delas, o método de Newton surge como uma das possibilidades. Acreditamos que a estrutura do texto não é usual, em textos introdutórios ou avançados sobre o tema. Aproveitamos a oportunidade para estimular a repensarmos as formas de apresentar conteúdos ou conceitos em matemática, porque acreditamos que a organização de resultados, ou a performance em um texto, sobre matemática pode dar maior ou menor impacto na compreensão e no interesse do leitor.

\section{INTRODUÇÃO}

Um raciocínio comum para aqueles que lidam com matemática, no meio das chamadas ciências exatas, envolve lidar com variáveis e, para alguns, é isso que amplia o conceito do que é matemática para além do conceito de lidar com números, ou de fazer aquilo que a calculadora do caixa de supermercado ou de alguma loja faz.

Um primeiro contato com variáveis ou incógnitas aparece quando os jovens alunos do Ensino Fundamental se deparam com equações como $x+2=4$ ou $x^{2}+2 x-1=0$. Mais adiante, talvez em cursos de graduação, alguns se deparam com equações como $3 \cos (x)=2, x^{5}+x-1=0$ ou uma mais interessante (ou complexa) $x^{2}+1=0$. Mas será que sabemos resolver tais equações? Bem, sem calculadora, muito poucas. E com calculadoras ou computadores, só algumas. Isso talvez motive alguns professores de matemática a dizerem que matemático não é calculadora, e de fato não é, e achamos que nem deveria tentar ser. Mas, certamente, alguns dos argumentos que contribuíram para o

Data de aceitação: Janeiro de 2021.

Palavras chave. Resolução numérica de equações, Método de Euler, Método de Newton, Otimização.

Agradeço ao parecerista pela leitura cuidadosa e pelos ótimos comentários e sugestões, que certamente melhoraram versão anterior deste texto. Agradeço aos professores José P. C. dos Santos e Nelson H. T. Lemes pela oportunidade de discutir e tentar pensar em questões envolvendo Análise Numérica e Equações Diferenciais. Agradeço minha esposa Rejane Siqueira Julio pelas conversas, pela companhia, pelo incetivo para tentar escrever e pelos nossos filhos Alice Julio Ferreira e Miguel Julio Ferreira que, de uma forma ou de outra, também contribuem com sua companhia entre pausas, brincadeiras e muitas outras boas coisas! 
funcionamento das calculadoras e dos computadores surgiram com a matemática, ou com algum uso dela.

Como as equações estão presentes em muitos problemas que surgem em aplicações e em teoria matemática é comum uma breve apresentação desse tema, em cursos de formação, para tratar de métodos para resolver equações, ou para aproximação de soluções, quando soluções existem.

Neste texto tratamos de duas formas de aproximação de soluções de equações, por meio do uso do método de Euler para a resolução numérica de equações diferenciais ordinárias que surgem em problemas de minimização. Em uma dessas formas o método de Newton surge como uma das possibilidades.

Os métodos de Euler e de Newton costumam ser apresentadas a discentes de cursos de graduação em matemática, computação e engenharia, por exemplo. Mas nem sempre são apresentados da forma que pretendemos aqui. Aproveitamos isso como uma oportunidade de mostrar que a matemática, seja lá o que isso for, não parece ser algo dividido em partes disjuntas, mas sim possuir intersecções importantes entre os mais diversos temas.

O problema que tentamos introduzir nesses breves comentários e o caminho que pretendemos seguir neste texto, pode, de certa forma, ser formalizado neste final de seção.

Antes de qualquer formalismo, alertamos o leitor que vamos usar símbolo em negrito para denotar vetores, como o vetor $\mathbf{u}$, e itálico para denotar números reais, como o número $t$.

Vamos denotar o módulo ou a norma euclidiana de um vetor por $\|\cdot\|$, que depende do produto interno usual (ou escalar) aqui denotado por $\langle\cdot, \cdot\rangle$. Quanto a matrizes $A_{m \times m}$, denotamos

$$
\|A\|=\max _{\|\mathbf{u}\|=1}\|A \mathbf{u}\|, \quad \mathbf{u} \in \mathbb{R}^{m} .
$$

Consideramos uma função $\mathbf{f}: U \rightarrow \mathbb{R}^{m}$, que por simplicidade supomos que possua derivadas contínuas até a ordem 2 , no conjunto aberto $U \subset \mathbb{R}^{m}$. Supomos ainda que a equação

$$
\mathbf{f}(\mathbf{u})=\left(f_{1}(\mathbf{u}), f_{2}(\mathbf{u}), \ldots, f_{m}(\mathbf{u})\right)=\mathbf{0}
$$

possua solução a $\in U$. Nesse caso, cada $f_{i}: U \rightarrow \mathbb{R}$ é uma função que possui derivada de segunda ordem contínua e tal que $f_{i}(\mathbf{a})=0$. Argumento semelhante pode ser aplicado à função

em que

$$
g(\mathbf{u})=\frac{1}{2}\|\mathbf{f}(\mathbf{u})\|^{2}, \quad \mathbf{u} \in U
$$

$$
0=g(\mathbf{a})=\min _{\mathbf{u} \in U} g(\mathbf{u})
$$

Isso mostra que a determinação de raízes de equações está ligado a problemas de otimização. O que, de certa forma, é sabido por quem já cursou disciplinas de Cálculo Diferencial, porque nessas disciplinas problemas de otimização estão relacionados ao problema de determinar as raízes da função gradiente de $g(\mathbf{u})$, por exemplo. É um tema antigo e que está ligado a muitos artigos recentes ([1, 2, 3, 4]); o tema vai e volta em meio a outros interesses que vão surgindo ([5, 6]).

Nesse ponto, cabe mencionar que a ideia de escrever este texto surgiu após leitura do artigo [2] e de perceber que a compreensão do tema tratado lá parecera-nos muito mais intuitiva se o texto fosse conduzido numa ordem diferente, e é isso que buscamos aqui. 


\section{A DERIVADA E A DERIVADA DIRECIONAL}

Lembramos rapidamente que a derivada da função $\mathbf{f}$, no ponto $\mathbf{u}$, é uma transformação linear que denotamos como $\mathbf{f}^{\prime}(\mathbf{u}): \mathbb{R}^{m} \rightarrow \mathbb{R}^{m}$, que melhor aproxima a variação da função $\mathbf{f}$, quando aplicada a pontos próximos de $\mathbf{u}$. Podemos também visualizar $\mathbf{f}^{\prime}(\mathbf{u})=J \mathbf{f}(\mathbf{u})$ como a matriz

$$
J \mathbf{f}(\mathbf{u})=\left[\begin{array}{llll}
\frac{\partial f_{1}(\mathbf{u})}{\partial u_{1}} & \frac{\partial f_{1}(\mathbf{u})}{\partial u_{2}} & \ldots & \frac{\partial f_{1}(\mathbf{u})}{\partial u_{m}} \\
\frac{\partial f_{2}(\mathbf{u})}{\partial u_{1}} & \frac{\partial f_{2}(\mathbf{u})}{\partial u_{2}} & \ldots & \frac{\partial f_{2}(\mathbf{u})}{\partial u_{m}} \\
\vdots & \vdots & \ddots & \vdots \\
\frac{\partial f_{m}(\mathbf{u})}{\partial u_{1}} & \frac{\partial f_{m}(\mathbf{u})}{\partial u_{2}} & \cdots & \frac{\partial f_{m}(\mathbf{u})}{\partial u_{m}}
\end{array}\right],
$$

cujas entradas são derivadas parciais de $f_{i}$, e que também é conhecida como matriz Jacobiana de $\mathbf{f}(\mathbf{u})$. A fim de simplificar a notação vamos considerar o vetor $\mathbf{u}$ como uma matriz coluna e $\mathbf{f}^{\prime}(\mathbf{u}) \mathbf{h}$ denotará a multiplicação da matriz $J \mathbf{f}(\mathbf{u})$ por $\mathbf{h}$, ou a aplicação da transformação linear $\mathbf{f}^{\prime}(\mathbf{u})$ no vetor $\mathbf{h}$, que é a derivada direcional de $\mathbf{f}$, no ponto $\mathbf{u}$ e na direção $\mathbf{h}([7,8,9])$.

Em resumo, vale a relação

$$
\mathbf{f}(\mathbf{u}+\mathbf{h})=\mathbf{f}(\mathbf{u})+\mathbf{f}^{\prime}(\mathbf{u}) \mathbf{h}+\mathbf{r}(\mathbf{h})
$$

com

$$
\lim _{\mathbf{h} \rightarrow 0} \frac{\|\mathbf{r}(\mathbf{h})\|}{\|\mathbf{h}\|}=0
$$

que é equivalente à definição da derivada de $\mathbf{f}$ no ponto $\mathbf{u}$.

Isso (ou a regra da cadeia) nos ajuda a calcular a derivada de $g$, no ponto u. Antes, observe que

$$
g(\mathbf{u})=\frac{1}{2}\|\mathbf{f}(\mathbf{u})\|^{2}=\frac{1}{2}\langle\mathbf{f}(\mathbf{u}), \mathbf{f}(\mathbf{u})\rangle,
$$

denotando o produto escalar usual de $\mathbb{R}^{m} \operatorname{como}\langle\cdot, \cdot\rangle$ ([9]). Segue então que

com

$$
\begin{aligned}
g(\mathbf{u}+\mathbf{h}) & =\frac{1}{2}\langle\mathbf{f}(\mathbf{u}+\mathbf{h}), \mathbf{f}(\mathbf{u}+\mathbf{h})\rangle \\
& =\frac{1}{2}\left\langle\mathbf{f}(\mathbf{u})+\mathbf{f}^{\prime}(\mathbf{u}) \mathbf{h}+\mathbf{r}(\mathbf{h}), \mathbf{f}(\mathbf{u})+\mathbf{f}^{\prime}(\mathbf{u}) \mathbf{h}+\mathbf{r}(\mathbf{h})\right\rangle \\
& =\frac{1}{2}\langle\mathbf{f}(\mathbf{u}), \mathbf{f}(\mathbf{u})\rangle+\left\langle\mathbf{f}^{\prime}(\mathbf{u}) \mathbf{h}, \mathbf{f}(\mathbf{u})\right\rangle+\mathbf{R}(\mathbf{h})
\end{aligned}
$$

O que nos diz que

$$
\lim _{\mathbf{h} \rightarrow \mathbf{0}} \frac{\|\mathbf{R}(\mathbf{h})\|}{\|\mathbf{h}\|}=0
$$

$$
g^{\prime}(\mathbf{u}) \mathbf{h}=\left\langle\mathbf{f}^{\prime}(\mathbf{u}) \mathbf{h}, \mathbf{f}(\mathbf{u})\right\rangle=\left\langle\mathbf{h},\left[\mathbf{f}^{\prime}(\mathbf{u})\right]^{*} \mathbf{f}(\mathbf{u})\right\rangle,
$$

em que $\left[\mathbf{f}^{\prime}(\mathbf{u})\right]^{*}$ denota a matriz adjunta (no caso, a transposta) da matriz $J \mathbf{f}(\mathbf{u})$, considerando $\mathbf{f}(\mathbf{u})$ também como matriz coluna.

Como $g(\mathbf{u})$ é um número real, é comum denotarmos

$$
g^{\prime}(\mathbf{u})=\left[\mathbf{f}^{\prime}(\mathbf{u})\right]^{*} \mathbf{f}(\mathbf{u})=\nabla g(\mathbf{u}),
$$


em que $\nabla$ representa o vetor gradiente, o que implica na igualdade

$$
g^{\prime}(\mathbf{u}) \mathbf{h}=\langle\mathbf{h}, \nabla g(\mathbf{u})\rangle,
$$

que é a derivada direcional de $g$, no ponto $\mathbf{u}$ e na direção $\mathbf{h}$.

Para uso seguinte, observamos que a Expressão (1) pode também ser escrita como

$$
\mathbf{f}(\mathbf{u}+\mathbf{h})=\mathbf{f}(\mathbf{u})+\int_{0}^{1} \mathbf{f}^{\prime}(\mathbf{u}+t \mathbf{h}) \mathbf{h} d t
$$

Isso produz de imediato a desigualdade do valor médio

$$
\|\mathbf{f}(\mathbf{u}+\mathbf{h})-\mathbf{f}(\mathbf{u})\| \leq \max _{0 \leq t \leq 1}\left\|\mathbf{f}^{\prime}(\mathbf{u}+t \mathbf{h})\right\|\|\mathbf{h}\| .
$$

2.1. A direção de maior decrescimento. Lembramos que a direção de maior decrescimento de $g(\mathbf{u})$, partindo de um ponto $\mathbf{u}_{0} \in U$, é dada pela direção oposta a do vetor gradiente $\nabla g\left(\mathbf{u}_{0}\right)$.

Isso define uma curva (ou caminho) $\mathbf{u}(t) \in U$, para $0 \leq t \leq T$, cuja derivada coincide com o oposto do vetor gradiente de $g$, no ponto $\mathbf{u}(t)$. Ou seja, $\mathbf{u}(t)$ é solução da equação diferencial ordinária $([10,11])$, ou problema de valor inicial,

$$
\left\{\begin{array}{l}
\mathbf{u}^{\prime}(t)=-\nabla g(\mathbf{u}(t)) \\
\mathbf{u}(0)=\mathbf{u}_{0}
\end{array},\right.
$$

o que quer dizer que $g(\mathbf{u}(t))$ é decrescente, desde que $\nabla g(\mathbf{u}(t)) \neq \mathbf{0}$.

No caso em que $\mathbf{u}_{0}$ é suficientemente próximo de $\mathbf{a}$, isso sugere que $\mathbf{u}(t)$ está ainda mais próximo de a, para $t>0$, o que melhora quando $t$ cresce ([1]). Um olhar mais atento para questões de unicidade da solução da Equação (6) sugere também que $\mathbf{u}(t)$ nunca atinge $\mathbf{a}$, a menos que $\mathbf{u}_{0}$ seja $\mathbf{a}$.

Nesse ponto, podemos pensar, bem, eu tinha uma equação $\mathbf{f}(\mathbf{u})=\mathbf{0}$ para resolver e agora tenho uma equação diferencial para resolver. Parece que a situação piorou um pouco! E isso tem como agravante o fato de não sabermos resolver a maioria das equações diferencias. Ou ainda o fato de equações diferenciais não ser um tema tratado de forma explícita em muitos cursos de graduação em áreas exatas. Mas acreditamos que isso mudaria se prestássemos mais atenção às leis de Newton e se permitíssemos e incentivássemos, em salas aula, mais conversas direcionadas aos motivos de estudarmos certos conceitos, como os relacionados com as disciplinas de Cálculo e de Física.

\section{O MÉTOdo DE EULER}

Nem sempre é possível determinar a função $\mathbf{u}(t)$ que é solução da Equação (6) e, por isso, vamos discutir como aproximar $\mathbf{u}(h), \mathbf{u}(2 h), \ldots, \mathbf{u}(p h)$, para algum número real $0<T$ e $p h=T$, para algum número natural $p$.

Como supomos que $\mathbf{f}(\mathbf{u})$ tem derivadas contínuas até a ordem 2 , então podemos usar $\mathbf{u}(t)$ e $\mathbf{u}^{\prime}(t)$ no lugar de $\mathbf{f}(\mathbf{u})$, nas expressões (1) e (4), para escrever a igualdade

$$
\mathbf{u}((j+1) h)=\mathbf{u}(j h)-\nabla g(\mathbf{u}(j h)) h+\tau_{j}(h),
$$

em que $\tau_{j}(h)$ é o erro de truncamento ([7, 11, 12]), que representa o erro cometido na aproximação

$$
\mathbf{u}((j+1) h) \approx \mathbf{u}(t h)-\nabla g(\mathbf{u}(j h)) h,
$$


sendo

$$
\left\|\tau_{j}(h)\right\| \leq M h^{2}
$$

Esse argumento produz o método de Euler para aproximar $\mathbf{u}(j h)$ como

$$
\begin{aligned}
\mathbf{u}(h) & \approx \mathbf{u}_{0}-h \nabla g\left(\mathbf{u}_{0}\right)=\mathbf{u}_{1} \\
\mathbf{u}(2 h) & \approx \mathbf{u}_{1}-h \nabla g\left(\mathbf{u}_{1}\right)=\mathbf{u}_{2} \\
\vdots & \vdots \\
\mathbf{u}(p h) & \approx \mathbf{u}_{p-1}-h \nabla g\left(\mathbf{u}_{p-1}\right)=\mathbf{u}_{p}
\end{aligned}
$$

Cabe aqui observar que, para obtermos uma aproximação de $\mathbf{u}(T)$, fazemos $p$ aproximações e que em cada uma delas pode haver um erro

$$
\mathbf{E}_{j}=\mathbf{u}\left(t_{j}\right)-\mathbf{u}_{j}, \quad 1<j \leq p,
$$

que certamente interfere na aproximação final.

Vamos estimar até que ponto os erros $\mathbf{E}_{j}$, em cada estimativa $\mathbf{u}_{j}$, podem interferir na aproximação $\mathbf{u}_{p} \approx \mathbf{u}(p h)$.

Da nossa hipótese sobre $\mathbf{f}(\mathbf{u})$ e da Expressão (5), diminuindo um pouco o conjunto $U$ e deixando-o convexo, se necessário, segue que

$$
\|\nabla g(\mathbf{u})-\nabla g(\mathbf{v})\| \leq L\|\mathbf{u}-\mathbf{v}\|, \quad \mathbf{u}, \mathbf{v} \in U
$$

para $L>0$, que depende da segunda derivada de $g$.

A Expressão (7), o método de Euler e a desigualdade (8) implicam que

$$
\begin{aligned}
\left\|\mathbf{E}_{j+1}\right\| & =\left\|\mathbf{u}\left(t_{j}\right)-\nabla g\left(\mathbf{u}\left(t_{j}\right)\right) h+\tau_{j}(h)-\left(\mathbf{u}_{j}-\nabla g\left(\mathbf{u}_{j}\right) h\right)\right\| \\
& \leq\left\|\mathbf{E}_{j}\right\|+h L\left\|\mathbf{E}_{j}\right\|+M h^{2},
\end{aligned}
$$

quando $h$ for suficientemente pequeno.

Repetindo o raciocínio, para $0<j<p-1$, obtemos

$$
\begin{aligned}
\left\|\mathbf{E}_{j+1}\right\| & \leq(1+h L)\left\|\mathbf{E}_{j}\right\|+M h^{2} \\
& \leq(1+h L)\left((1+h L)\left\|\mathbf{E}_{j-1}\right\|+M h^{2}\right)+M h^{2} \\
\vdots & \vdots \vdots \\
& \leq\left((1+h L)^{j}+\cdots+(1+h L)+1\right) M h^{2} \\
& =\frac{(1+h L)^{j+1}-1}{h L} M h^{2} \\
& <\left(e^{T L}-1\right) \frac{M}{L} h
\end{aligned}
$$

As duas últimas desigualdades seguem da soma dos termos de uma progressão geométrica e do fato de que $1+h L<e^{h L}$, para $0<h$.

Esse raciocínio para estimar $\left|\mathbf{E}_{j}\right|$ mostra que este depende linearmente de $h$ e demonstra o lema a seguir $([10,11,12])$. Em particular, segue que, se $T$ for suficientemente grande, $\mathbf{u}_{0}$ for suficientemente próximo de a e $h$ for suficientemente pequeno, então (a menos de limitação computacional) $\mathbf{u}_{p}$ será uma boa aproximação para $\mathbf{a}$. 
Lema 3.1. Considere a equação diferencial

$$
\left\{\begin{array}{l}
\mathbf{u}^{\prime}(t)=\mathbf{F}(t, \mathbf{u}(t)) \\
\mathbf{u}(0)=\mathbf{u}_{0}
\end{array}, \quad 0 \leq t \leq T,\right.
$$

com $\mathbf{F}$ contínua e tal que

$$
\|\mathbf{F}(t, \mathbf{u})-\mathbf{F}(t, \mathbf{v})\| \leq L\|\mathbf{u}-\mathbf{v}\| .
$$

Existe única solução $\mathbf{u}(t)$ para a Equação (9), para $0 \leq t \leq T$.

Ainda, se

$$
\left\|\frac{1}{2} \frac{d \mathbf{F}(t, \mathbf{u}(t))}{d t}\right\| \leq M
$$

$e$

$$
\mathbf{u}_{j+1}=\mathbf{u}_{j}+h \mathbf{F}\left(j h, \mathbf{u}_{j}\right), \quad 0 \leq j \leq p-1,
$$

então

$$
\left\|\mathbf{u}(j h)-\mathbf{u}_{j}\right\| \leq\left((1+h L)^{j+1}-1\right) \frac{M}{L} h<\left(e^{T L}-1\right) \frac{M}{L} h .
$$

O erro que pode ocorrer em cálculos aproximados, causados pela precisão da calculadora que usarmos, é importante, mas não vamos nos ater a isso aqui. Esse erro tende a diminuir cada vez mais, com o passar do tempo ou com o avanço da tecnologia (se puder veja $[12,13])$.

O Lema 3.1 é bastante importante em análise de métodos numéricos. Mas como podemos ver, é resultado geral, e não diz algo sobre a estimativa de $\left\|\mathbf{u}_{p}-\mathbf{a}\right\|$, que é o que nos interessa nessa discussão. Será que a forma da Equação (6) pode nos mostrar algo nessa linha?

\section{Controlando A Velocidade DE CONVERGÊNCIA}

A forma de determinar uma aproximação para $\mathbf{a}$, tal que $\mathbf{f}(\mathbf{a})=\mathbf{0}$, por meio da direção de maior decrescimento de $g(\mathbf{u})$ e do método de Euler, parece que pode ser bastante lenta, porque o erro que estimamos é proporcional a $h$, e não sabemos o quão perto $\mathbf{u}(T)$ está de a.

Sendo assim, procuramos determinar agora se é possível, a partir de certo ponto, estimar a velocidade de convergência de $\mathbf{u}_{j}$ para a, ou mesmo escolher outra curva $\mathbf{u}(t)$ em que $g(\mathbf{u}(t))$ tenha um decrescimento que conseguimos controlar ou estimar.

Para termos uma ideia de possíveis caminhos a escolher observe o argumento que segue. Este raciocínio está relacionado com teoria de controle e de funcionais de Lyapunov (se puder veja $[1,2,3,6]$ ).

A dedução da Expressão (3) e a regra da cadeia nos ajudam a concluir que

$$
\begin{aligned}
\frac{d g(\mathbf{u}(t))}{d t} & =g^{\prime}(\mathbf{u}(t)) \mathbf{u}^{\prime}(t) \\
& =\left\langle\mathbf{u}^{\prime}(t), \nabla g(\mathbf{u}(t))\right\rangle \\
& =\left\langle\mathbf{u}^{\prime}(t),[J \mathbf{f}(\mathbf{u}(t))]^{*} \mathbf{f}(\mathbf{u}(t))\right\rangle \\
& =\left\langle J \mathbf{f}(\mathbf{u}(t)) \mathbf{u}^{\prime}(t), \mathbf{f}(\mathbf{u}(t))\right\rangle
\end{aligned}
$$


Supondo que $\mathbf{u}(t)$ é solução da Equação (6) e usando a desigualdade de Cauchy-Schwarz concluímos então que

$$
\begin{aligned}
\frac{d g(\mathbf{u}(t))}{d t} & =-\langle\nabla g(\mathbf{u}(t)), \nabla g(\mathbf{u}(t))\rangle \\
& =-\left\langle[J \mathbf{f}(\mathbf{u}(t))]^{*} \mathbf{f}(\mathbf{u}(t)),[J \mathbf{f}(\mathbf{u}(t))]^{*} \mathbf{f}(\mathbf{u}(t))\right\rangle \\
& =-\left\|[J \mathbf{f}(\mathbf{u}(t))]^{*} \mathbf{f}(\mathbf{u}(t))\right\|^{2} \\
& \leq-\alpha(t)\|\mathbf{f}(\mathbf{u}(t))\|^{2} \\
& =-2 \alpha(t) g(\mathbf{u}(t))
\end{aligned}
$$

sendo $0 \leq \alpha(t)$ o menor autovalor de matriz $[J \mathbf{f}(\mathbf{u}(t))]^{*} J \mathbf{f}(\mathbf{u}(t))$, ou seja $\sqrt{\alpha(t)}$ é o menor valor singular de $J \mathbf{f}(\mathbf{u}(t))$ ([9]).

Nos argumentos a seguir aproveitamos para apresentar uma equação diferencial que pode ser resolvida. Por isso usamos $g(t)$ no lugar de $g(\mathbf{u}(t))$, e podemos supor que $\alpha(t)$ é uma função contínua qualquer. Definimos

$$
\lambda(t)=\int_{0}^{t} \alpha(s) d s
$$

Seja $r:[0, T] \rightarrow[0,+\infty)$ uma função contínua e tal que

$$
g^{\prime}(t)+2 \alpha(t) g(t)+r(t)=0 .
$$

Então, pelas regras do produto e da cadeia,

$$
\begin{aligned}
\frac{d\left(e^{2 \lambda(t)} g(t)\right)}{d t} & =e^{2 \lambda(t)} g^{\prime}(t)+2 \alpha(t) e^{2 \lambda(t)} g(t) \\
& =-e^{2 \lambda(t)} r(t)
\end{aligned}
$$

Integrando ambos os lados da igualdade e usando o Teorema Fundamental do Cálculo temos que

$$
e^{2 \lambda(t)} g(t)-g(0)=-\int_{0}^{t} e^{2 \lambda(s)} r(s) d s .
$$

Como $0 \leq r(t)$, concluímos que

$$
0 \leq \int_{0}^{t} e^{2 \lambda(s)} r(s) d s
$$

e que

$$
g(t) \leq g(0) e^{-2 \lambda(t)}
$$

Isso demonstra o lema que segue.

Lema 4.1. Seja u $\mathbf{u}(t)$ uma solução da Equação (6) e $0 \leq \alpha(t)$ o menor autovalor de matriz $[J \mathbf{f}(\mathbf{u}(t))]^{*} J \mathbf{f}(\mathbf{u}(t))$. Então

$$
g(\mathbf{u}(t)) \leq g\left(\mathbf{u}_{0}\right) e^{-2 \int_{0}^{t} \alpha(s) d s} .
$$

No caso em que a matriz $J \mathbf{f}(\mathbf{a})$ for invertível, existe um número real $0<\alpha<\alpha(t)$ e o lema anterior pode ser reformulado como segue. 
Lema 4.2. Nas consições Lema 4.1, se Jf $(\mathbf{a})$ for invertível e $\mathbf{u}_{0}$ for suficientemente próximo de a. Então existe $0<\alpha$ tal que

$$
g(\mathbf{u}(t)) \leq g\left(\mathbf{u}_{0}\right) e^{-2 \alpha t} .
$$

Isso nos diz que a escolha da direção de maior decrescimento nos dá uma curva $\mathbf{u}(t)$ que, em tese, nos permite aproximar a de tal forma $g(\mathbf{u}(t))$ tenha decaimento exponencial, desde que $J \mathbf{f}(\mathbf{a})$ seja invertível. Em tempos de pandemia principalmente, é conhecido que esse decaimento é rápido. Mas determinar o valor de $\alpha$ pode ser uma tarefa complicada, afinal queremos descobrir o valor de a, que supomos existir, mas que não conhecemos.

Por outro lado, como geralmente precisamos aproximar $\mathbf{u}(t)$ numericamente, uma outra pergunta interessante é a seguinte: Será que, nas condições do Lema 4.1, é possível que o método de Euler para resolver a Equação (6), com $\mathbf{u}_{0}$ suficientemente próximo de a, seja tal que $\left\|\mathbf{u}_{j}-\mathbf{a}\right\|$ tenha decaimento exponencial?

A resposta dessa pergunta é sim, e o curioso é que $J \mathbf{f}(\mathbf{a})$ precisa apenas ser uma matriz não nula. Se for esse o caso, então $J \mathbf{f}(\mathbf{u})$ será também não nula para todo $\mathbf{u} \in U \mathrm{e}$ suficientemente próximo de a, digamos, para todo $\mathbf{u}$ tal que $\|\mathbf{u}-\mathbf{a}\|<r$, para algum $0<r$.

Para nos auxiliar, definimos a função $\phi$ como

$$
\phi(\mathbf{u})=\mathbf{u}-h[J \mathbf{f}(\mathbf{u})]^{*} \mathbf{f}(\mathbf{u}), \quad\|\mathbf{u}-\mathbf{a}\|<r .
$$

Sendo assim, se $\left\|\mathbf{u}_{0}-\mathbf{a}\right\|<r$ e $\mathbf{u}_{j}$ for obtido pelo método de Euler, lembrando que $\nabla g(\mathbf{u})=[J \mathbf{f}(\mathbf{u})]^{*} \mathbf{f}(\mathbf{u})$, então

$$
\mathbf{u}_{j+1}=\phi\left(\mathbf{u}_{j}\right) .
$$

Como $f(\mathbf{a})=\mathbf{0}$, temos ainda que $\phi(\mathbf{a})=\mathbf{a}$.

Isso nos leva a observar que o método de Euler toma a forma de um método de ponto fixo (se puder veja $[12,14]$ ).

Usando a regra da cadeia e a regra do produto obtemos a igualdade

$$
\phi^{\prime}(\mathbf{u})=I-h\left[(J \mathbf{f}(\mathbf{u}))^{\prime}\right]^{*} f(\mathbf{u})-h[J \mathbf{f}(\mathbf{u})]^{*} J \mathbf{f}(\mathbf{u})
$$

em que $I$ denota a matriz identidade.

Seja

$$
S^{*} S=I, \quad D=\left[\begin{array}{cccc}
s_{1}^{2} & 0 & \cdots & 0 \\
0 & s_{2}^{2} & \cdots & 0 \\
\vdots & \vdots & \ddots & \vdots \\
0 & 0 & \cdots & s_{m}^{2}
\end{array}\right], \quad S^{*} D S=[J \mathbf{f}(\mathbf{a})]^{*} J \mathbf{f}(\mathbf{a})
$$

a decomposição em autovalores e autovetores da matriz $[J \mathbf{f}(\mathbf{a})]^{*} J \mathbf{f}(\mathbf{a})$ ([9]). Segue que $\|S\|=\left\|S^{*}\right\|=1$ e que

$$
\begin{aligned}
\left\|\phi^{\prime}(\mathbf{a})\right\| & =\left\|I-h[J \mathbf{f}(\mathbf{a})]^{*} J \mathbf{f}(\mathbf{a})\right\| \\
& =\left\|S^{*}(I-h D) S\right\| \\
& \leq\|I-h D\| \\
& =\max _{1 \leq i \leq m}\left|1-h s_{i}^{2}\right|
\end{aligned}
$$


Supondo que $s_{m} \leq \cdots \leq s_{2} \leq s_{1}$, podemos escolher $0<h<2 s_{1}^{-2}$ e obtermos

$$
\left\|\phi^{\prime}(\mathbf{a})\right\|=N_{0}<N<1 \text {. }
$$

Diminuindo $r$, se necessário, a desigualdade do valor médio implica que

$$
\|\phi(\mathbf{u})-\phi(\mathbf{v})\| \leq N\|\mathbf{u}-\mathbf{v}\|, \quad\|\mathbf{u}-\mathbf{a}\|<r, \quad\|\mathbf{v}-\mathbf{a}\|<r .
$$

Em particular, se $\left\|\mathbf{u}_{0}-\mathbf{a}\right\|<r$, então

$$
\begin{aligned}
\left\|\mathbf{u}_{j+1}-\mathbf{a}\right\| & =\left\|\phi\left(\mathbf{u}_{j}\right)-\phi(\mathbf{a})\right\| \\
& \leq N\left\|\mathbf{u}_{j}-\mathbf{a}\right\| \\
& =N\left\|\phi\left(\mathbf{u}_{j-1}\right)-\phi(\mathbf{a})\right\| \\
& \leq N^{2}\left\|\mathbf{u}_{j-1}-\mathbf{a}\right\| \\
& \vdots \\
& \vdots \\
& \leq N^{j+1}\left\|\mathbf{u}_{0}-\mathbf{a}\right\| \\
& \leq e^{-(j+1) \beta}\left\|\mathbf{u}_{0}-\mathbf{a}\right\|
\end{aligned}
$$

em que $e^{-\beta}=N$, o que nos dá o decaimento exponencial para a $\left\|\mathbf{u}_{j}-\mathbf{a}\right\|$, e demonstra o teorema que segue. Observe que nos argumentos anteriores poderíamos ter tomado $h$ variável em cada iteração, mantendo a restrição $\left|1-h s_{1}^{2}\right|<N<1$.

Teorema 4.3. Se a matriz Jf $(\mathbf{a})$ for não nula e $\left|1-h s_{1}^{2}\right|<N<1$, então existe $0<r$, tal que

$$
\left\|\mathbf{u}_{0}-\mathbf{a}\right\|<r \Longrightarrow\left\|\mathbf{u}_{j}-\mathbf{a}\right\| \leq e^{-j \beta}\left\|\mathbf{u}_{0}-\mathbf{a}\right\|
$$

em que $e^{-\beta}=N$, quando $\mathbf{u}_{j}$ for dado pelo método de Euler para resolver a Equação (6).

Porém, como não conhecemos a, não temos como conhecer $s_{1}$ e escolher $h$ que nos dê o decaimento estimado para $\left\|\mathbf{u}_{j}-\mathbf{a}\right\|$, o que nos leva a seção seguinte.

4.1. O método de Newton. Com a resposta que obtemos nos lemas 4.1 e 4.2, nos perguntamos se é possível escolher antes um valor para $\alpha$ e encontrar um caminho $\mathbf{u}(t)$ que satisfaça a Desigualdade (11)?

No ponto em que estamos, a resposta vem rápido. Segue da Expressão (10) que

$$
\frac{d g(\mathbf{u}(t))}{d t}=\left\langle J \mathbf{f}(\mathbf{u}(t)) \mathbf{u}^{\prime}(t), \mathbf{f}(\mathbf{u}(t))\right\rangle
$$

o que demonstra o resultado que queremos e apresentamos agora.

Lema 4.4. Seja $\alpha>0$. Se u(t) for solução da equação

$$
\left\{\begin{aligned}
J \mathbf{f}(\mathbf{u}(t)) \mathbf{u}^{\prime}(t) & =-\alpha \mathbf{f}(\mathbf{u}(t)) \\
\mathbf{u}(0) & =\mathbf{u}_{0}
\end{aligned}\right.
$$

então

Em particular,

$$
\frac{d g(\mathbf{u}(t))}{d t}=-2 \alpha g(\mathbf{u}(t))
$$

$$
g(\mathbf{u}(t))=g\left(\mathbf{u}_{0}\right) e^{-2 \alpha t}
$$


Em tese, obtemos uma nova equação diferencial, dada pela Expressão (12), que nos permite controlar a velocidade em que $g(\mathbf{u}(t))$ converge para 0 . Mas como ocorre com a Equação (6) pode ser necessário o uso de um método numérico para a aproximação da solução dessa nova equação.

Supondo que a matriz $J \mathbf{f}(\mathbf{a})$ seja invertível e que $\mathbf{u}_{0}$ esteja suficientemente próximo de a, podemos mais uma vez aplicar o método de Euler para aproximar a (única) solução da Equação (12), que pode agora ser escrita como

$$
\left\{\begin{array}{l}
\mathbf{u}^{\prime}(t)=-\alpha[J \mathbf{f}(\mathbf{u}(t))]^{-1} \mathbf{f}(\mathbf{u}(t)) \\
\mathbf{u}(0)=\mathbf{u}_{0}
\end{array} .\right.
$$

Para evitarmos o cálculo da matriz inversa de $J \mathbf{f}(\mathbf{u}(t))$, escrevemos o método de Euler como

$$
\left\{\begin{array}{rl}
J \mathbf{f}\left(\mathbf{u}_{j}\right) \mathbf{w}_{j} & =-h \alpha \mathbf{f}\left(\mathbf{u}_{j}\right) \\
\mathbf{u}_{j+1} & =\mathbf{u}_{j}+\mathbf{w}_{j}
\end{array},\right.
$$

em que $\mathbf{u}\left(t_{j+1}\right) \approx \mathbf{u}_{j+1}, t_{j+1}=t_{j}+h$, sendo $0<h$.

$\mathrm{O}$ restante da seção nos mostra que essa nova forma de determinar $\mathbf{u}_{j}$ traz alguma melhora em relação a escolha de $h$ e quanto a convergência de $\mathbf{u}_{j}$, quando comparamos com o Teorema 4.3. Os argumentos que usaremos são quase idênticos aos que usamos antes, por isso apresentamos aqui apenas o que achamos que difere um pouco.

Se $J \mathbf{f}(\mathbf{a})$ for invertível, então $J \mathbf{f}(\mathbf{u})$ será também invertível, quando $\|\mathbf{u}-\mathbf{a}\|<r$, para algum $0<r$, e podemos definir a função

$$
\psi(\mathbf{u})=\mathbf{u}-h \alpha[J \mathbf{f}(\mathbf{u})]^{-1} \mathbf{f}(\mathbf{u}), \quad\|\mathbf{u}-\mathbf{a}\|<r .
$$

A Expressão (13) implica que

$$
\mathbf{u}_{j+1}=\psi\left(\mathbf{u}_{j}\right),
$$

caso $\left\|\mathbf{u}_{0}-\mathbf{a}\right\|<r$, e, mais uma vez, vemos um método de ponto fixo relacionado ao método de Euler, agora para outra equação diferencial, pois

$$
\mathbf{f}(\mathbf{a})=\mathbf{0}, \quad \psi(\mathbf{a})=\mathbf{a} .
$$

Ainda,

$$
\begin{aligned}
\psi^{\prime}(\mathbf{u}) & =I-h \alpha\left[(J \mathbf{f}(\mathbf{u}))^{\prime}\right]^{-1} \mathbf{f}(\mathbf{u})-h \alpha[J \mathbf{f}(\mathbf{u})]^{-1} J \mathbf{f}(\mathbf{u}) \\
& =(1-h \alpha) I-h \alpha\left[(J \mathbf{f}(\mathbf{u}))^{\prime}\right]^{-1} \mathbf{f}(\mathbf{u})
\end{aligned}
$$

e

$$
\left\|\psi_{j}^{\prime}(\mathbf{a})\right\|=|1-h \alpha| .
$$

Escolhendo $0<h$, tal que $0 \leq|1-h \alpha| \leq N<1$, e repetindo argumentos anteriores, obtemos a demonstração do teorema que segue, o que nos dá novamente um decaimento exponencial para $\left\|\mathbf{u}_{j}-\mathbf{a}\right\|$, mas agora podemos escolher $h$ de forma clara. Em particular, a restrição imposta a $h$ não impede que o mesmo varie, em cada iteração.

Teorema 4.5. Se a matriz Jf $(\mathbf{a})$ for invertivel e $|1-\alpha h| \leq N<1$, então existe $0<r$, tal que

$$
\left\|\mathbf{u}_{0}-\mathbf{a}\right\|<r \Longrightarrow\left\|\mathbf{u}_{j}-\mathbf{a}\right\| \leq e^{-j \beta}\left\|\mathbf{u}_{0}-\mathbf{a}\right\|
$$

em que $e^{-\beta}=N$, quando $\mathbf{u}_{j}$ for dado pela Expressão (13). 
Quanto melhor for a escolha de $\mathbf{u}_{0}$, menor pode ser $N$ no teorema anterior. A convergência tende a ser cada vez mais rápida a cada iteração, porque $\mathbf{u}_{j}$ pode ser visto como condição inicial para a próxima iteração.

Em tese, podemos ainda tomar $N$ tão pequeno quanto quisermos, quando escolhemos $h \alpha=1$, o que produz o método de Newton para resolver a equação $\mathbf{f}(\mathbf{u})=\mathbf{0}$. Sob o ponto de vista prático, de certa forma, isso desmotiva a escolha de $\alpha \neq 1$ na Equação (12), caso tenha a intenção de usar o método de Euler. Entretanto, tendo em mente o Lema 3.1, a escolha de $h$ menor pode aumentar a região de escolha de $\mathbf{u}_{0}$ em que $\mathbf{u}_{j}$ converge para a.

O Lema 3.1 sugere que o método de Euler pode ser usado inicialmente para aproximar o valor de $\mathbf{u}_{p} \approx \mathbf{u}(T) \approx \mathbf{a}$, usando a Equação (6), que é computacionalmente mais simples que a Equação (12). Depois disso, se desconfiarmos que $J \mathbf{f}(\mathbf{a})$ possui inversa, podemos usar a Expressão (13) para calcular $\mathbf{u}_{j}$, para $j>p$, para tentar convergência mais rápido para a. Mas se desconfiarmos que $J \mathbf{f}(\mathbf{a})$ não possui inversa, ou que está próxima disso, o Teorema 4.3 pode ser reconfortante.

4.2. Outras possibilidades. Uma outra possibilidade para tentar aumentar a velocidade de convergência de $\mathbf{u}_{j}$, dado pelo método de Euler, para a solução da equação $\mathbf{f}(\mathbf{u})=\mathbf{0}$, é não se preocupar muito com a curva $\mathbf{u}(t)$, que é solução de alguma equação diferencial, e deixar $h$ variar em cada iteração, com algum critério para que $g\left(\mathbf{u}_{j+1}\right)<g\left(\mathbf{u}_{j}\right)$ (veja $[2,3])$.

Podemos também usar outros métodos numéricos para resolver as equações diferenciais que aparecem neste texto e, com isso, diminuir o erro que cometemos nas aproximações $([11,12])$. Por simplicidade, vamos descrever o caso do método de Euler Modificado, que é dado por

$$
\mathbf{u}((j+1) h) \approx \mathbf{u}_{j+1}=\mathbf{u}_{j}+h \mathbf{u}^{\prime}\left(\mathbf{u}_{j}+\frac{h}{2} \mathbf{u}^{\prime}\left(\mathbf{u}_{j}\right)\right) .
$$

No caso da Equação (12), por exemplo, esse método pode ser aplicado em duas etapas. Obtemos primeiro uma estimativa $\tilde{\mathbf{u}}_{j}$ como

$$
\tilde{\mathbf{u}}_{j+1}=\mathbf{u}_{j}+\frac{h}{2} \mathbf{u}^{\prime}\left(\mathbf{u}_{j}\right) \Longrightarrow\left\{\begin{array}{rl}
J \mathbf{f}\left(\mathbf{u}_{j}\right) \tilde{\mathbf{w}}_{j} & =-\frac{h}{2} \alpha \mathbf{f}\left(\mathbf{u}_{j}\right) \\
\tilde{\mathbf{u}}_{j+1} & =\mathbf{u}_{j}+\tilde{\mathbf{w}}_{j}
\end{array},\right.
$$

sendo $\tilde{\mathbf{u}}_{0}=\mathbf{u}_{0}$, e depois obtemos $\mathbf{u}_{j}$ como

$$
\mathbf{u}_{j+1}=\mathbf{u}_{j}+h \mathbf{u}^{\prime}\left(\tilde{\mathbf{u}}_{j}\right) \Longrightarrow\left\{\begin{array}{rl}
J \mathbf{f}\left(\tilde{\mathbf{u}}_{j}\right) \mathbf{w}_{j} & =-h \alpha \mathbf{f}\left(\tilde{\mathbf{u}}_{j}\right) \\
\mathbf{u}_{j+1} & =\mathbf{u}_{j}+\mathbf{w}_{j}
\end{array} .\right.
$$

Entretanto, esse método é computacionalmente mais caro que o método de Euler. Mesmo assim, podemos usar argumentos da demonstração do Teorema 4.5 para mostrar que a convergência desse último método é mais rápida que a do método de lá, mas deixamos isso para o leitor.

É possível manipular a Expressão (4) para obter métodos numéricos que têm semelhanças com o que discutimos neste texto ([6]). Se o leitor tiver interesse em outros tipos de métodos, ou em estimativas de custo computacional, ou desejar evitar o cálculo de derivadas em suas implementações computacionais, sugerimos, por exemplo, a leitura do texto [4] e referências. 


\section{COMENTÁRIOS FINAIS}

Para finalizar este texto, observamos que os teoremas 4.3 e 4.5 sugerem que a equação $\mathbf{f}(\mathbf{u})=\mathbf{0}$ pode ainda ser resolvida por meio da equação diferencial

$$
\left\{\begin{array}{l}
\mathbf{u}^{\prime}(t)=-A(\mathbf{f}(\mathbf{u}(t)) \mathbf{f}(\mathbf{u}(t)) \\
\mathbf{u}(0)=\mathbf{u}_{0}
\end{array}\right.
$$

em que $A(\mathbf{f}(\mathbf{u}(t)))$ é uma matriz que faz com que $g(\mathbf{u}(t))$ tenha decaimento controlado, ou estimado, de alguma forma. $\mathrm{O}$ uso de método numéricos para aproximar $\mathbf{u}(t)$, dado pela Equação (14), nos dá vislumbres para criar vários métodos de ponto fixo para aproximar a solução da equação $\mathbf{f}(\mathbf{u})=\mathbf{0}$.

Como exemplo particular, seja $\mathbf{f}(\mathbf{u})=B \mathbf{u}-\mathbf{v}$, para alguma matriz $B_{n \times n}$ e algum vetor $\mathbf{v}$ conhecido. Escolha $A\left(\mathbf{f}(\mathbf{u}(t))=[\operatorname{diag}(B)]^{-1}\right.$, sendo $\operatorname{diag}(B)$ a matriz cuja diagonal principal é igual a de $B$ e com zeros nas demais entradas. Então o método de Euler, para resolver numericamente a versão da Equação (14), nesse caso, inclui o método de ponto fixo de Jacobi-Richardson, para resolver a equação $B u=v$, quando $h=1$. Se, em vez disso, tomarmos $A\left(\mathbf{f}(\mathbf{u}(t))=[\operatorname{diag}(B)+L]^{-1}\right.$, sendo $\operatorname{diag}(B)+L$ a matriz cujos termos acima da diagonal principal são nulos e os demais são iguais aos de $B$. Então o método de Euler, com $h=1$, é agora o método de ponto fixo de Gauss-Seidel.

\section{REFERÊNCIAS}

[1] Y. C. Huang, S. H. Sun, J. Q. Han, Neural networks based on Lagrange multiplier for nonlinear programming, Acta Electron. Sin. 26, 24-28, 1998.

[2] L. Zhou, Y. Wu, L. Zhang, G. Zhang, Convergence analysis of a differential equation approach for solving nonlinear programming problems, Applied Mathematics and Computation, Volume 184, Issue 2, 789-797, 2007. https://doi.org/10.1016/j.amc.2006.05.190

[3] I. M. Ross, An optimal control theory for nonlinear optimization, Journal of Computational and Applied Mathematics, Volume 354, 39-51, 2019. https://doi.org/10.1016/j.cam.2018. 12.044

[4] J. R. Sharma, D. Kumar, On a reduced cost derivative-free higher-order numerical algorithm for nonlinear systems. Comp. Appl. Math. 39, 202, 2020. https://doi.org/10.1007/ s $40314-020-01218-3$

[5] J. C. Ferreira, M. C. Baquião, A least square point of view to reproducing kernel methods to solve functional equations, Applied Mathematics and Computation, 2019. https://doi.org/10.1016/ j.amc.2019.04.008.

[6] C. A. Tavares, T. M. R. Santos, N. H.T. Lemes, J. P.C. Santos, J. C. Ferreira, J. P. Braga, Solving ill-posed problems faster using fractional-order Hopfield neural network, Journal of Computational and Applied Mathematics, Volume 381, 2021. https://doi.org/10.1016/j.cam.2020. 112984

[7] E. L. Lima, Análise Real, Vol. 2, Rio de Janeiro, Instituto Nacional de Matemática Pura e Aplicada, 2004.

[8] G. B. Thomas, Cálculo, Vol. 2, Pearson, Ed. 11, 2009.

[9] H. P. Bueno, Álgebra Linear: Um segundo curso, Rio de Janeiro, Sociedade Brasileira de Matemática, 2006.

[10] D. G. Zill, Equações diferenciais com aplicações em modelagem. 3. ed., São Paulo: Cengage Learning, 2016.

[11] D. J. Lambert, Numerical Methods for Ordinary Differential Systems: The Initial Value Problem, John Wiley \& Sons, Inc., USA, 1991.

[12] R. L. Burden, J. D. Faires, Análise Numérica, São Paulo, Cengage Learning, 2008. 
[13] D. S. Clark, Short proof of a discrete gronwall inequality, Discrete Applied Mathematics, Volume 16, Issue 3, 279-281, ISSN 0166-218X, 1987. https : / / doi .org/10 .1016/0166-218X (87) $90064-3$

[14] A. B. M. Brunner, P. R. S. Malta, Teoremas de ponto fixo de Banach e Knaster-Tarski, revistos, Revista Matemática Universitária, vol. 2, 2020. https://doi .org/10.21711/26755254/ rmu202021

UNIVERSIDADE FEDERAL DE ALFENAS

Email address: jose.ferreira@unifal-mg.edu.br 